\title{
NOTULAE SYSTEMATICAE
}

Tome XII, FASCICUles 1 et 2 (MARS I945)

\section{DESCRIPTIONS DE NOUVELLES ACANTHACEES MALGACHES}

par R. BENOIST

\section{Ruellia transitoria sp. nov.}

Frutex ramosus, ramis junioribus pubescentia alba densa, pilis glandulosis mixta, vestitis, vetustioribus glabratis, cortice griseo indutis. Folia petiolata, petiolo dense pubescente cum pilis glandulosis brevibus, ovato-lanceolata, basi acuta, apice obtusa, margine subintegro vel saepius crenulato-repando, pagina utraque dense pubescente, pilis brevibus glandulosis intermixtis. Flores solitarii, axillares, unusquisque bracteolis duabus linearibus sepalorum dimidiam longitudinem aequantibus praeditus. Sepala 5 subaequalia, linearia, usque ad basin libera, pubescentia, pilis glandulosis brevibus intermixtis. Corollae roseo-violaceae tubus basi breviter cylindricus, superne usque ad faucem ampliatus; lobi ovati, parum inaequales. Stamina 4 didynama, ad medium tubi corollae inserta, filamentis gracilibus, glabris, duobus cujusque lateris basi longe concrescentibus ; pollinis granula sphaerica, alveolata. Ovarium dense pubescens, ovula circiter 6 in quoque loculo gerens; stylus pilosus ; stigma bilabiatum, labio uno brevissimo, altero in laminam elongatam producto. Capsula subquadrangularis, fere fusiformis, pubescens, semina 5-6 in quoque loculo gerens.

Pétiole long de 8-12 mm. ; feuilles longues de $15-20 \mathrm{~mm}$., larges de 7-12 mm. ; sépales longs de Io- $12 \mathrm{~mm}$.; corolle longue de $33 \mathrm{~mm}$., son tube long de $20 \mathrm{~mm}$.; capsule longue de $\mathrm{r} 2 \mathrm{~mm}$.

Madagascar : domaine du sud-ouest : baie de Saint-Augustin, fleur rose violacé, I ${ }^{\text {er }}$ juin 1939 (Decary, I4.II6).

Le pollen est alvéolé, les parois des alvéoles très peu hautes semblent formées de granulations disposées en lignes et chacune concrescente avec ses voisines.

\section{Ruellia patula Jacq.}

BALFour (Flora of Socotora, p. 209) cite cette espèce de Madagascar. Il s'agit sans doute du Ruellia cyanea Bojer qui est très proche du $R$. patula. 


\section{Ruellia singalaris sp. nov.}

Herba erecta, parum ramosa, ramis junioribus minute puberulis, mox glabris. Folia breviter petiolata, cujusque paris inaequalia, lanceolata, basi acuta, apice breviter acuminata, margine integro vel aliquando obscure repando, pagina utraque primum pilis sparsis albidis cito deciduis ornata, superiore denique glabra, inferiore in nervis pilosula. Flores axillares, solitarii (an semper ?), sessiles. Sepala 5, inaequalia, foliacea, margine sinuato, posticum ovato-lanceolatum majus, lateralia sublinearia minora, omnia ad basin libera. Corollae violaceae tubus a basi ad faucem sensim ampliatus ; lobi 5 ovati, parum inaequales. Stamina 4 didynama, circiter ad quintam parten inferiorem tubi inserta, duo cujusque lateris filamentorum basi concrescentia, filamentis glabris; antheris apice dentibus duobus armatis ; pollinis granula sphaerica, tuberculis rotundatis ornata. Discus cupuliformis. Ovarium glabrum, apice pubescens, ovula $5-6$ in quoque loculo gerens ; stylus glaber ; stigma in laminam ovato-rhomboideam expansum.

Pétiole long de $2 \mathrm{~mm}$.; feuilles longues de $4-8 \mathrm{~cm}$., larges de II-25 mm. ; sépale postérieur long de I5 mm., large de $8 \mathrm{~mm}$. ; sépales latéraux longs de $9 \mathrm{~mm}$., larges de I mm. ; corolle longue de $22 \mathrm{~mm}$., son tube long de $16 \mathrm{~mm}$.

Madagascar : bois près de la rivière Anove sur la côte orientale, corolle violacée (Perrier de la Bâthie 9.443).

\section{Ruellia gruicollis R. Benoist. \\ var. angustifolia var. nov.}

A speciminibus typicis differt foliis lanceolato-linearibus, $20-25 \mathrm{~mm}$. longis, 4-5 mm. latis.

Madagascar : grès de l'Isalo vers $800 \mathrm{~m}$. d'altitude, juillet Igro (Perrier de la Bâthie 4.99I).

\section{Ruellia Perrieri sp. nov.}

Frutex ramosus, ramis junioribus breviter albo-pubescentibus, subtetragonis, vetustioribus cylindricis glabris, cortice griseo, internodiis neatim attenuata et in crassiuscula, breviter petiolata, oblonga, basi cumargine integro, pagina utolo decurrentia, apice obtusa vel rotundata, cellati, ad pedicelli basintraque glabra. Flores axillares, solitarii, pediminute albo-fimbriatis praediti. Calicis brevibus lanceolatis in margine dium concrescentia, parte libera late segmenta 4 glabra usque ad mebriato, antico breviter bilibera late ovata, margine minute albo-fim- 
dricus, hinc usque ad faucem sensim ampliatus, modice curvatus ; lobi 5 parum inaequales, ovati, apice subbilobi. Stamina 4 didynama, ad quartam partem inferiorem tubi corollae inserta, filamentis glabris, satis crassis, basi insertione discretis; pollinis granula sphaerica reticulatoalveolata. Ovarium glabrum ovula circiter 8 in quoque loculo gerens; stylus glaber. Capsula ignota.

Feuilles longues de $15-16 \mathrm{~mm}$., larges de 5-6 mm. ; pédicelle floral long de $5 \mathrm{~mm}$. environ; bractéoles longues de $2 \mathrm{~mm}$. ; calice long de 6-7 mm., partie libre des sépales large de 4-4,5 $\mathrm{mm}$. ; corolle longue de $35 \mathrm{~mm}$., son tube long de $23 \mathrm{~mm}$.

Madagascar : domainle du sud-ouest, plateau calcaire Mahafaly sur la rive gauche du Menarandra, arbuste rameux de I-2 mètres, corolle d'un rouge vif, juin I9Io (Perrier de la Bathie 9.490).

Cette plante est remarquable par son calice dont les sépales antérieurs sont concrescents presque jusqu'au sommet, les autres l'étant seulement jusqu'au milieu ; une concrescence analogue des sépales existe aussi chez d'autres espèces malgaches, en particulier chez $R$. albopurpurea $R$. Ben. et $R$. latisepala $R$. Ben. De plus les étamines sont insérées isolément, celles d'un même côté n'étant pás concrescentes par la base des filets. Cependant malgré ces différences c'est un véritable Ruellia.

\section{Ruellia prostrata Poiret.}

var. rivularis Boivin mss. var. nov.

A speciminibus typicis differt internodiis $\mathrm{r}_{5-20} \mathrm{~mm}$. longis, foliis anguste lanceolatis, $26-60 \mathrm{~mm}$. longis, $8-16 \mathrm{~mm}$. latis; sepalis $6 \mathrm{~mm}$. longis; corolla $20 \mathrm{~mm}$. longa.

Madagascar: Nossi-Bé, bords du ruisseau d'Andradroit, mars 185I (Boivin).

\section{Strobilanthes curviflora sp. nov.}

Frutex ramis junioribus tetragonis, in faciebus sulcatis, parum pilosis, mox glabris, vetustioribus teretibus, cortice griseo. Folia petiolata, lanceolata vel ovato-lanceolata, basi attenuata et in petiolo decurrentia, apice acuminata, margine integro, pagina utraque breviter et sparse pilosula, costa nervos secundarios 7-8 utrinque gerente. Flores in spicis 
brevibus paucifloris, pedunculatis, axillaribus vel in ramis vetustioribus, foliis jam delapsis, dispositi. Bracteae foliaceae, parum dense pilosoglandulosae, basi in petiolum angustatae ; bracteolae lineari-spatulatae, sparse pilosae. Sepala 5 parum inaequalia, usque ad basin libera, linearia, acuta, apice pilosa. Corollae tubus basi curvatus, a basi ad faucem sensim ampliatus, lobi fere aequales, ovati, apice emarginati. Stamina 4 didynama parum infra medium tubi inserta ; filamentis pilosis, duobus cujusque lateris basi concrescentibus; pollinis granula sphaerica, echinulata. Discus annuliformis sub ovario situs. Ovarium maxima parte glabrum, extremo apice pilosum, ovula 2 in quoque loculo gerens ; stylus pilosus ; stigma in laminas duas lineares, valde inaequales bipartitum. Capsula ovoideo-compressa, glabra.

Pétiole long de $2-5 \mathrm{~cm}$. ; feuilles longues de $8-16 \mathrm{~cm}$., larges de 38-60 mm. ; bractéoles longues de I5 mm. environ; larges de $3 \mathrm{~mm}$. vers l'extrémité ; sépales longs de $9 \mathrm{~mm}$., larges de I mm. ; corolle longue de $35 \mathrm{~mm}$., son tube long de $28 \mathrm{~mm}$. ; capsule longue de II-I2 mm.

Madagascar : massif de l'Andrangovalo au sud-est du lac Alaotra, réserve naturelle $\mathrm{n}^{0} 3$ dite de Zakamena, dans le bassin de l'Onibe, forêt ombrophile vers $1200 \mathrm{~m}$. (Humbert et Cours I7.95I).

\section{Strobilanthes cuneifolia sp. nov.} Herba erecta, ramosa, ramis junioribus tetragonis glabris, in faciebus
sulcatis. Folia lanceolata, basi longe attenuata, in petiolo alato pro-
tracta, apice acuminata, tracta, apice acuminata, margine integro, pagina superiore glabra, infedarios Io-12 utrinquargine pilis minutis ornata, costa nervos secunaxillaribus, breviter peduncute. Flores in spicis brevibus paucifloris, basi in petiolum angedunculatis dispositi. Bracteae foliaceae, glabrae, in margine pilis paucistae ; bracteolae lineari-spatulatae fere glabrae, libera, linearia, glabra. Corollae tubus 5 subaequalia, usque ad basin sensim ampliatus, lobi inaequales basi curvatus, a basi ad faucem ginati vel parum incisi. Staminales, ovato-triangulares, apice emartubi inserta, filamentis pilosis, 4 didynama parum supra medium tibus ; pollinis granula pilosis, duobus cujusque lateris basi concrescenovario situs. Ovarium sphaerica, echinulata. Discus annuliformis sub stylus pilosus; stigma in laminas ovula duo in quoque loculo gerens ; sula ignota.

Feuilles longues de 7 - $17 \mathrm{~cm}$., larges de $18-40 \mathrm{~mm}$., pédoncule de l'inflorescence long de 5-12 mm. ; bractées longues de 12 - 
I $8 \mathrm{~mm}$., larges de $3-8 \mathrm{~mm}$. ; bractéoles longues de $7 \mathrm{~mm}$. ; sépales longs de $7 \mathrm{~mm}$., larges de $0,75 \mathrm{~mm}$. ; corolle longue de $22 \mathrm{~mm}$., son tube long de $\mathrm{I} 7 \mathrm{~mm}$.

Madagascar : massif du Tsaratanana, à I.700 m. d'alt., forêt à sous-bois herbacé, dans les endroits plutôt secs (cimes, crêtes, etc.) où cette plante forme des taches sporadiques mais étendues; grande plante bisannuelle de $I$ à 2 mètres, très rameuse ; fleurs blanches à gorge veinée largement de rouge (Perrier de la Bâthie 15.270).

\section{Strobilanthes ramiflora sp. nov.}

Suffrutex ramis junioribus tetragonis in faciebus sulcatis, parce pilosis, deinde glabris. Folia petiolata, lanceolata, basi in petiolo longe decurrentia, apice acuminata, margine integro, pagina utraque pilis sparsis in nervis ornata, costa nervos secundarios 6-7 utrinque gerente. Flores in spicis axillaribus et terminalibus parum elongatis, saepe compositis dispositi ; spicis pedunculo circiter I $5 \mathrm{~mm}$. longo praeditis, earum axi tetragono parce et adpresse albo-piloso. Bracteae oppositae, foliaceae, foliis similes sed minores. Flores sessiles bracteolis 2 linearibus in margine pilis albis satis longis praęditi. Sepala 5 aequalia lineari-lanceolata, acuta, in margine longe albo-pilosa. Corollae albae tubus curvatus, a basi ad faucem sensim ampliatus, lobi 5 parum inaequales, intus albo-pilosi. Stamina 4 didynama ad medium tubum inserta, filamentis pilosis, duobus cujusque lateris basi concrescentibus; pollinis granula sphaerica echinata. Discus annuliformis sub ovario situs. Ovarium glabrum ovula 2 in quoque loculo gerens ; stylus pilosus ; stigma bilabiatum, labio uno brevi, altero in laminam linearem elongato. Capsula ignota.

Pétiole long de $2-3 \mathrm{~cm}$. f feuille longue de $7-\mathrm{I}_{3} \mathrm{~cm}$., larges de 3-4 cm. ; épis longs de $2-6 \mathrm{~cm}$., leurs entrenœuds longs de I2I $8 \mathrm{~mm}$. ; bractéoles longues de $9 \mathrm{~mm}$., larges de I mm. ; sépales longs de 6-7 mm. ; corolle longue de $3 \mathrm{~cm}$., son tube long de $22 \mathrm{~mm}$.

Madagascar : bois, mont Angavokely, I800 m. d'alt. ; plante sous-frutescente souvent cauliflore, haute de I m. $50 \mathrm{~cm}$. à 2 mètres, à fleur blanche (Perrier de la Bâthie I6.075).

\section{Strobilanthes breviceps sp. nov.}

Herba ramosa caulibus basi saepe procumbentibus, ramis junioribus tetragonis, in faciebus sulcatis, parum dense pilosis. Folia petiolata, 
ovato-lanceolata, basi in petiolo decurrentia, apice acuminata, margine integro, pagina utraque parum dense pilosa, superiore viridi, inferiore pallidiore, costa nervos secundarios circiter ro utrinque emittente. Flores in spicis brevibus, densis, capitatis, breviter pedunculatis, axillaribus dispositi ; pedunculis pilosis. Bracteae foliaceae rufescenti-pilosae. Sepala 5 subaequalia linearia, usque ad basin libera, apice obtusiuscula, rufescenti-pilosa. Corollae roseae extus pilosae tubus basi breviter cylindricus, superne sensim usque ad faucem ampliatus, lobi ovati subaequales. Stamina 4 didynama vix infra medium tubum inserta, filamentis pilosis, duobus cujusque lateris basi concrescentibus ; pollinis granula sphaerica echinulata. Discus annuliformis sub ovario situs. Ovarium glabrum ovula duo in quoque loculo gerens; stylus pilosus; stigma in laminas duas lineares inaequales partitus. Capsula ignota.

Pétiole long de $\mathrm{I}-4 \mathrm{~cm}$.; feuilles longues de $8-\mathrm{I} 8 \mathrm{~cm}$., larges de 35-75 mm. ; pédoncule de l'inflorescence long de 5-I5 mm. ; bractées longues de $2-4 \mathrm{~cm}$., larges de 6 -II mm. ; bractéoles longues de I2-I3 mm., larges de 2,5-3 mm. ; sépales longs de Io $\mathrm{mm}$., larges de I mm. ; corolle longue de $55 \mathrm{~mm}$., son tube long de $42 \mathrm{~mm}$.

Madagascar : forêt orientale humide, près de la rivière Anove, à l'alt. de $200 \mathrm{~m}$. ; plante herbacée de $60 \mathrm{~cm}$. à I m. $20 \mathrm{~cm}$., rameuse ; tige souvent couchée à la base ; corolle rose vif, septembre IgIa (Perrier de la Bâthie 9.460), type.

Je rapporte à la même espèce l'échantillon suivant :

Forêt à sous-bois herbacé d'Andanibe dans le bassin de l'Onive vers I. 400 mètres d'alt. ; plante sous-frutescente à la base, rameuse, 2-3 mètres de haut ; fleurs roses, novembre I9II (Perrier de la Bâthie 9.303).

\section{Strobilanthes mediocris sp. nov.}

Suffrutex ramis junioribus tetragonis, rufo-pilosis, deinde subcylindricis glaberscentibus. Folia petiolata, lanceolata, basi longe attenuata, gina utraque acuminata, apice ipso obtusiusculo, margine integro, pacundarios 6-7 utrin dense pilosa, inferiore pallidiore, costa nervos sevibus, ad flores 2 oppositas. Inflorescentiae in spicis axillaribus, brebracteolae lineari-spatulatae, rufo-pilosae. 2 oppositas reductis. ad basin libera, linearia, apice rufo-pilosae. Sepala 5 subaequalia, usque bus ad faucem sensim, apice obtusa, pilosa. Corollae sparse pilosae tumina 4 didynama ad dilatatus, lobi 5 ovati, parum inaequales. Stacujusque lateris basi concrescentibum inserta, filamentis pilosis, duobus pollinis granula sphaerica, ochi- 
nulata. Discus annuliformis sub ovario situs. Ovarium glabrum, ovula 2 in quoque loculo gerens. Capsula ignota.

Pétiole long de 8-I2 mm. ; feuilles longues de 4,5-10 cm., larges de I5-26 mm. ; pédoncule des inflorescences long de 6-Io mm., bractéoles longues de $7 \mathrm{~mm}$. ; larges de I;5 mm. ; sépales longs de 7-8 mm., larges de $0,75 \mathrm{~mm}$. ; corolle longue de $32 \mathrm{~mm}$., son tube long de $23 \mathrm{~mm}$.

Madagascar : mont Tsaratanana, forêt à sous-bois herbacé, $2.000 \mathrm{~m}$. d'altitude; grande plante sous-ligneuse, fleur blanche à palais rose (Perrier de la Bâthie I5.589), type. - Massif du Tsaratanana et haute vallée du Sambirano (réserve naturelle $n^{\circ} 4$ ), forêt ombrophile sur sol siliceux, plante suffrutescente de 5-8 décimètres, corolle blanche avec nervures et petites macules rouges, vers $2.000 \mathrm{~m}$. (Humbert I8.277).

L'échantillon suivant semble appartenir à la même espèce ; ses feuilles sont plus larges et mesurent $8-\mathrm{I} 7 \mathrm{~cm}$. sur 3,5-6 cm.

Montagnes entre le haut Sambirano et le haut Maivarano (entre Mangindrano et Ampanompia, forêt ombrophile sur latérite de gneiss, corolle blanche veinée de rouge (Humbert I8.II3).

\section{Mimulopsis angustata sp. nov.}

Herba ramis subquadrangularibus parum dense piloso-glandulosis Folia petiolata, ovata, basi cordiformia, apice acuminata, margine irregulariter et aliquando grosse dentato vel subintegro, pagina utraque sparse pilosa. Inflorescentiae terminales, axi bracteas oppositas, unas inferiores foliis similes, alteras superiores foliaceas lanceolatas sensim decrescentes, sparse pilosas gerente ; ad axillam cujusque bracteae cyma pauciflora piloso-glandulosa orta. Flores pedicellati, pedicello pilosoglanduloso. Sepala 5 usque ad basin libera parum inaequalia linearia, obtusa, piloso-glandulosa. Corollae roseae vel intense rubrae tubus curvatus, a basi ad faucem obliquam modice ampliatus ; lobi 5 subaequales, ovati. Stamina 4 didynama in tubo corollae ad quartam partem superiorem inserta, duobus cujusque lateris basi filamentorum sparse pilosorum concrescentibus; staminum anteriorum antheris loculo uno calcarato, altero mutico; posteriorum autem antheris loculo utroque mutico. Ovarium basi glabrum, apice pubescens ovula 4 in quoque loculo gerens ; stylus pilosus ; stigma bifidum, ramis valde inaequalibus. Capsula basi glabra, apice pubescens. 
Pétiole long de $2-7 \mathrm{~cm}$. ; feuilles longues de $7-16 \mathrm{~cm}$., larges de 4-II cm. ; inflorescence longue de $I 5-20 \mathrm{~cm}$. ; pédicelles longs de 5-6 mm. ; calice long de 9-1o $\mathrm{mm}$. ; corolle longue de 26 $30 \mathrm{~mm}$., son tube long de $20 \mathrm{~mm}$. ; capsule longue de 9-10 $\mathrm{mm}$.

Madagascar : massif de Manongarivo vers I.200 m. forêt centrale à sous-bois herbacé (Perrier de la Bâthie 9.245).

\section{Mimulopsis armata sp. nov.}

Herba ramosa, caulibus quadrangularibus glabris. Folia petiolata, ovata vel ovato-lanceolata, basi subcordiformia, apice acuminata, margine irregulariter dentato, pagina superiore praeter nervos glabra, inferiore glabra. Inflorescentiae terminales, earum axi bracteas oppositas, foliaceas, lanceolatas, superne magis magisque decrescentes gerente ; unaquaeque bractearum ad axillam cymam laxam paucifloram emittens. Flores breviter pedicellati. Sepala 5 usque ad basin libera, linearia, parum inaequalia piloso-glandulosa. Corollae rubrae tubus a basi ad faucem modice ampliatus ; lobi 5 , subaequales, oblongi. Stamina 4 didynama ad tertiam partem inferiorem tubi inserta, duo cujusque lateris basi filamentorum concrescentia ; filamentis in parte inferiore pilis paucis ornatis ; antherae omnes basi bicalcaratae; pollinis granula ellipsoidea longitudinaliter sulcata. Ovarium glabrum ovula 4 in quoque loculo gerens ; stylus fere glaber, pilis raris sparsis ornatus ; stigma bifidum ramis 2 valde inaequalibus. Capsula glabra, apice acuta.

Pétiole long de 4-Io centimètres ; feuilles longues de 6-I4 cm., larges de $4-8 \mathrm{~cm}$. ; pédicelles longs de $\mathrm{I}-3 \mathrm{~mm}$. ; calice long de IO-I $3 \mathrm{~mm}$.; corolle longue de $3 \mathrm{~cm}$., son tube long de $2 \mathrm{~cm}$. ; capsule longue de $\mathrm{I} 8 \mathrm{~mm}$.

Madagascar : forêt de Didy (Catat I.734).

\section{Mimulopsis Catati sp. nov.}

Frutex (?) ramis subquadrangularibus glabris. Folia petiolata, cujusque paris parum inaequalia, ovata, basi modice cordiformia, apice tiae terminalusa, margine integro, pagina utraque glabra. Inflorescen-

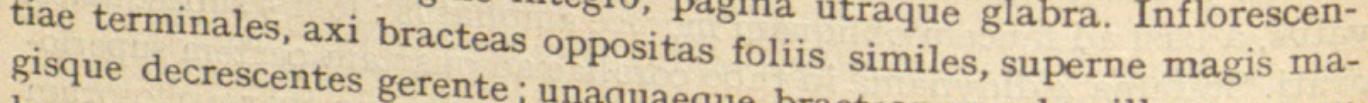
laxam paucifloram emittens ; cymarum bractearum ad axillam cymam glabris ; flores breviter pedicellati. Sepacteis lanceolatis, sessilibus, nearia, subacuta, glabra, parum in Sepala 5 usque ad basin libera, livatus, a basi ad faucem sensim inaequalia. Corollae luteae tubus curapice breviter incisi. Stamina 4 didynama, lobi 5 , subaequales, ovati, inferiorem tubi inserta, duo cujusquema parum supra quartam partem 
rum concrescentia ; antherarum anteriorum loculo uno calcarato, altero mutico, posteriorum autem antheris muticis; pollinis granula ellipsoidea longitudinaliter sulcata. Ovarium et stylus pubescentes ; stigma bifidum, ramis valde inaequalibus.

Pétiole long de 3-I6 mm. ; feuilles longues de I6-55 mm., larges de 9-35 mm. ; bractées des cymes longues de 5-7 mm.; pédicelle floral long de $2 \mathrm{~mm}$. ; calice long de I2-I4 mm.; corolle longue de $3 \mathrm{~cm}$., son tube long de $2 \mathrm{~cm}$.

Madagascar : Nossi-Ve (Catat I.230).

\section{Forsythiopsis linifolia sp. nov.}

Frutex ramosus, ramis junioribus glabris, sulcis 2 oppositis notatis, vetustioribus teretibus, cortice griseo. Folia sessilia, linearia, acuta, glabra. Flores solitarii, axillares, pedicellati, pedicello bracteas duas minutas supra basin gerente. Sepala 5 aequalia, linearia, acuta, usque ad basin libera, glabra. Corollae lilacinae bilabiatae tubus subcylindricus, labium superius bilobum, inferius trilobum, lobis oblongis, obtusis. Stamina 2 fertilia antica, unumquodque cum staminodio (laterali) concrescens, filamentis glabris, antheris bilocularibus. Ovarium glabrum ; stylus sparse pilosus. Capsula glabra.

Feuilles longues de 8-10 mm. ; pédicelle long de 3-6 mm. ; sépales longs de $2 \mathrm{~mm}$. ; corolle longue de $\mathrm{r} 2 \mathrm{~mm}$., son tube long de $5 \mathrm{~mm}$. ; capsule jeune longue de $\mathrm{I} 2 \mathrm{~mm}$.

Madagascar : bassin de la Linta, plateau calcaire à l'Est du delta, alt. 200-250 m. ; fleurs lilas (Humbert et Swingle 5.507).

\section{Achyrocalyx pungens sp. nov.}

Frutex ramis junioribus pubescentia brevi albida vestitis. Folia breviter petiolata, linearia vel lanceolato-linearia, basi sensim angustata, apice acuta et spinescentia, margine integro vel aliquando ad basin spina acuta armato, subtus valde involuto, pagina superiore glabra, inferiore albo-tomentosa. Inflorescentiae breves, terminales, 4-6 florae, earum axi bracteas oppositas gerente. Bracteae lanceolatae, apice acuto spinescente, trinerviae. Sepala 5 , inaequalia, posterius majus, lateralia minora, omnia lanceolata, apice acuta fere spinescentia, usque ad basin libera. Corollae aurantiacae bilabiatae tubus a basi usque ad faucem modice et sensim ampliatus, labio superiore bilobo, inferiore trilobo. Stamina 4 didynama, ad tertiam partem inferiorem tubi inserta ; antheris unilocularibus. Ovarium glabrum. 
Feuilles à pédicelles longs de $2-3 \mathrm{~mm}$. ; limbe long de $2-4 \mathrm{~cm}$., large de $3-5 \mathrm{~mm}$. ; inflorescences longues de $2 \mathrm{~cm}$. environ; bractées et bractéoles longues de Io mm., larges de 3 I/4-3 I/2 $\mathrm{mm}$. ; sépale postérieur long de I2 mm. ; corolle longue de I3 mm., son tube long de $7 \mathrm{~mm}$.

Madagascar: bois calcaires (éocène) du plateau de Miandraraha dans le bassin du Manombo ; arbuste de $50 \mathrm{~cm}$. à I m. ; sommet des bractées et corolle oranges, mai IgIo (Perrier de la Bâthie 9.507).

\section{Neuracanthus brachystachyus sp. nov.}

Frutex ramis junioribus teretibus, primum pilosis, deinde fere glabris. Folia petiolata, lanceolata, basi satis repente attenuata et in petiolo denervis parrentia apice breviter acuminata, margine integro, pagina utraque in e spicis duabusa. Inflorescentiae axillares, valde breves, spicatae, saepe ceolatas, acutas, constitutae ; inflorescentiae axi bracteas oppositas, lancosta nervos laterales nullae. Calicis bilabiati extus que emittente, omnes fertiles ; bracteolae ticum tridentatum, dentes I mm. longi, parum ultra medium fissa, pos bilabiatae tubus subcylindricus, longi, omnium dentes acuti. Corollae inferius trilobum extus longe pilosis superius bilobum basi intus pilosum, lobis omnibus riorum antheris biamina 4 filamentis brevibus, sub fauce inserta, antenium connectivo transverse elongato gerente ; pollinis granula elongato ad quamque extremitatem loculum cum in quoque lo more generis. Ovarium glabrum ovulum unigum. Capsula compressa glens ; stylus glaber ; stigma unilaterale, oblonapice solida et sterilis.

Pétiole long de 8-I2 mm. ; feuilles longues de 5-7 cm., larges de $2-2,5 \mathrm{~cm}$. ; inflorescences longues d'environ I $\mathrm{cm}$. ; bractées longues de $7 \mathrm{~mm}$., larges de $2 . \mathrm{mm}$. ; calice long de $6 \mathrm{~mm}$. ; corolle longue de $6 \mathrm{~mm}$., son tube long de $4 \mathrm{~mm}$. ; capsule longue
de $6-7 \mathrm{~mm}$.

Madagascar : bords du Manombo (Ambongo), bois rocailleux sur basalte, avril I904 (Perrier dela Bâthie I7I9), type. - Bords de la baie de Diego Suarez, dans les broussailles, mai 1906 (Perrier de la Bâthie 9.35I) ; bois rocailleux calcaires de Kamakama sur le causse d'Ankara, juin Igor (Perrier de la Bâthie r.309). 


\section{Neuracanthus Leandrii sp. nov.}

Suffrutex, ramis junioribus subquadrangularibus, primum pubescentia arachnoidea sparse vestitis, deinde glabris. Folia petiolata, lanceolata, basi attenuata et in petiolo decurrentia, apice acuminata, margine integro, pagina superiore glabra, inferiore in nervis arachnoideo-pubescente. Inflorescentiae axillares, valde breves spicatae, axi bracteas oppositas, lanceolatas vel ovato-lanceolatas, acutas, concavas, extus parce arachnoideo-pilosas gerente. Bracteae quinquenerviae, omnes fertiles ; bracteolae nullae. Calicis bilabiati, extus pilis paucis ornati, labia fere usque ad basin distincta, posticum tridentatum, dentibus I $\mathrm{mm}$. longis, anticum bidentatum, dentibus $2 \mathrm{~mm}$. longis, dentibus omnibus acutis. Corollae glabrae, albae, bilabiatae, tubus subcylindricus, sub fauce parum constrictus ; labium inferius trilobum, superius bilobum. Stamina 4 filamentis brevibus, ad quartam partem superiorem tubi inserta, anteriorum antheris bilocularibus, posteriorum loculo postico obsoleto, omnium connectivo piloso ; pollinis granula sphaerica, laevia. Ovarium glabrum ovulum unicum in quoque loculo gerens ; stylus glaber; stigma unilaterale, oblongum.

Pétiole long de 4-8 mm. ; feuilles longues de 5-12 cm., larges de I $8-36 \mathrm{~mm}$. ; inflorescences longues de $7 \mathrm{~mm}$., larges de $3 \mathrm{~mm}$. ; calice long de $6 \mathrm{~mm}$. ; corolle longue de $8 \mathrm{~mm}$., son tube long de $5 \mathrm{~mm}$.

Madagascar : Tsingy du Bemaraha, Tsiandro, sur les rochers calcaires à l'alt. de $600 \mathrm{~m}$., nom vernaculaire : soasompa (Leandri 835) ; Tsingy du Bemaraha : Antsingy ( $9^{\circ}$ réserve) sur rochers calcaires en lisière de forêt, à l'alt. de 200 m., sous-arbrisseau à fleurs blanches (Leandri I.oI8).

\section{Ecbolium fimbriatum sp. nov.}

Suffrutex ramis junioribus glabris, longitudinaliter striatis. Folia sessilia, basi cordata, apice acuta, margine integro, glabra. Inflorescentiae terminales in spicis strobiliformibus densis; bracteae oppositae, omnes fertiles, sessiles, ovatae, acutae pilosae, margine integro, longius fimbriato. Flores bracteolis 2 linearibus pilosis. Sepala 5 aequalia, ad basin libera, linearia, piloso-glandulosa. Corollae violaceae bilabiatae tubus angustus, elongatus, cylindricus, labio superiore lineari, apice obtuso brevissime inciso, inferiore trilobato, lobis lateralibus oblongis, medio latiore elliptico. Stamina 2 sub fauce corollae inserta, filamentis glabris, antheris bilocularibus. Ovarium pubescens ovula 2 in quoque loculo gerens; stylus filiformis apice brevissime bifidus. Capsula clavata, 4 semina gerens. 
Feuilles longues de I5-20 mm., larges de II-I7 mm. ; bractées longues de $20 \mathrm{~mm}$., larges de 9-12 mm. ; bractéoles longues de I4 mm. ; sépales longs de I $2 \mathrm{~mm}$. ; corolle longue de $50 \mathrm{~mm}$., son tube long de $38 \mathrm{~mm}$.; capsule longue de $15 \mathrm{~mm}$.

Madagascar ; province de Tuléar, la Table, sur le calcaire ; fleur violette, 29 mars I92I (Poisson, $2^{8}$ voyage, I65).

\section{Sphacanthcs Humberti sp. nov.}

Herba caulibus junioribus quadrangularibus glabris. Folia petiolata cujusque paris parum inaequalia, lanceolata, basi attenuata apice acuminata, margine integro, pagina superiore glabra, inferiore in nervis parce pilosula, utraque cystolithis notata. Inflorescentiae terminalis axis bracteas oppositas parvas lanceolatas gerens; in cujusque bracteae, axilla nascitur cyma perbrevis $3-5$ flora; bracteolae bracteis similes. Sepala 5 inaequalia, ovata vel ovato-lanceolata, basi breviter concrescentia, apice breviter acuminata, glabra, aestivatione imbricata. Corollae bilabiatae tubus in tertia parte inferiore cylindricus, hinc sensim dilatatus, labium superius bilobum, inferius trilobum.Stamina 2 in tertia parte inferiore tubi inserta, filamentis glabris ; antheris bilocularibus; pollinis granula subsphaerica, parum compressa poris 2 oppositis area circulari granulosa circumdatis praedita. Ovarium glabrum ovula duo in quoque loculo gerens; stylus filiformis; stigma in laminas duas oblongas divi-
sum.

Pétiole long de $3-25 \mathrm{~mm}$.; feuilles longues de $3-8 \mathrm{~cm}$., larges de 10-32 mm. ; inflorescences longues de 5-9 cm. ; bractées longues de $2-3 \mathrm{~mm}$., larges de $0,75 \mathrm{~mm}$.; sépales longs de 5-6 mm., larges de $2 \mathrm{~mm}$. ; corolle longue de $20 \mathrm{~mm}$., son tube long de
$9 \mathrm{~mm}$.

Madagascar : forêt d'Analafanja, au nord du Fiherenana, sur plateau calcaire, altitude 950-I.000 m. (Humbert I4.289).

\section{Populina Perrieri sp. nov.} Frutex ramosus, ramis patulis. Folia decidua, petiolata, ovalia, basi
rotundata vel subtruncata, apice acuminata, margine integro saepe undu-
lato, pagina superiore glabra, inferiore in lato, pagina superiore glabra, inferiore in nervis parce pilosa. Infloresfertiles, ovatales in spicis parvis paucifloris ; bracteae oppositae, omnes nullae. Sepala 5 aequalia, lae, extus in costa parce pilosae ; bracteolae nuiter pubescentia. Corollae roseacuta, fere usque ad basin libera, teelongatus, labium superius obleae bilabiatae tubus cylindricus parum 
tripartitum. Stamina 2 sub fauce inserta, filamentis glabris, antheris bilocularibus, loculis aequalibus aequaliter alto insertis, basi minutissime mucronatis. Ovarium glabrum ; styli basis pubescens. Capsula basi sterilis et angustata, parte fertili ovoideo-compressa, apice acuta, loculo quoque semen unum discoideum, planum, tegumento minute granuloso gerens.

Feuilles (jeunes) longues de I0-25 mm., larges de 8-15 mm. ; bractées longues de 5-7 mm., larges de 3-4 mm. ; sépales longs de $4 \mathrm{~mm}$.; corolle longue de $16 \mathrm{~mm}$., son tube long de $7 \mathrm{~mm}$. ; capsule longue de $9 \mathrm{~mm}$.

Madagascar : rocailles (calcaire éocène) près de la mer à Majunga (Perrier de la Bâthie I2.898).

\section{Forcipella longistaminea sp. nov.}

Frutex ramis junioribus tenuiter puberulïs, longitudinaliter striatis. Folia decidua, petiolata, breviter ovata, basi obtusa et in medio in petiolo decrescentia, apice rotundata, margine integro, glabra. Flores in axillis superioribus ramorum solitarii, unusquisque breviter pedicellatus, bracteolis 2 ovatis obtusis minute pubescentibus suffultus. Sepala 5 aequalia, fere usque ad basin libera, lanceolato-linearia, apice subacuta, pilis brevibus glandulosis capitatis, sparse vestita. Corollae dilute purpureae bilabiatae tubus curvatus modice et sensim superne ampliatus, labium superius ovatum breviter bilobum, inferius profundius trilobum. Stamina 2 antica fertilia, antheris bilocularibus, 3 alia sterilia, antheris minutis, omnia exserta, filamentis valde elongatis, ad tertiam partem inferiorem tubi inserta. Discus cupuliformis ovarii basin circumcingens. Ovarium glabrum ovula duo in quoque loculo gerens; stylus glaber, elongatus ; stigma capitatum breviter bilobum.

Feuilles (jeunes) longues de $\mathbf{I} \mathbf{2} \mathbf{~ m m}$., larges de $8 \mathrm{~mm}$. ; bractéoles longues de $3 \mathrm{~mm}$., larges de $2 \mathrm{~mm}$., sépales longs de $5 \mathrm{~mm}$. ; corolle longue de $22 \mathrm{~mm}$., son tube long de $16 \mathrm{~mm}$.

Madagascar : plateau Mahafaly (Menarondra) sur les éboulis calcaires, juin rgIo (Perrier de la Bâthie 9.522).

\section{Hypoestes warpurioides sp. nov.}

Frutex ramis junioribus quadrangularibus, pilosis. Folia petiolata, petiolo tenuiter pubescente, lanceolata, basi attenuata, apice acuminata, acuta vel obtusiuscula, margine integro, pagina superiore pilis brevibus sparsis, in nervo principali numerosioribus, ornata, inferiore sparse pi- 
losa. Inflorescentiae axillares spicatae, pedunculo nudo, piloso, satis longo simplici vel trifurco praeditae ; spicae unilaterales breves densae ; bracteae oppositae, lanceolatae, pilosae, quadrifariae, quarum series 2 fertiles et 2 steriles, in axilla involucrum gerentes. Involucra e quatuor foliolis decussatis formata; foliolae exteriores lanceolato-lineares, ante apicem repente sed modice angustatae pilosae, in dimidio superiore pilis glandulosis vestitae interiore lanceolata-lineares, acutae, extus pilosae cum paucis pilis glandulosis in parte apicali. Sepala 5 subaequalia, usque fere ad basin libera, linearia, acuta, pilosa. Corollae roseae bilabiatae tubus a basi sensim et modice ampliatus, labio superiore subrhomboidali, inferiore trilobo. Stamina 2 ad faucem inserta, filamentis brevibus, antheris unilocularibus. Ovarium glabrum ; stylus sparse pilosus.

Pétiole long de 6-13 mm. ; feuille longue de 6-I3 cm., large de I3-24 mm. ; pédoncule de l'inflorescence long de $2-7 \mathrm{~cm}$. ; bractées longues de $8 \mathrm{~mm}$., larges de $3 \mathrm{~mm}$.; folioles externes de l'involucre longues de $8 \mathrm{~mm}$., larges de $3 \mathrm{~mm}$.; les internes longues de $7 \mathrm{~mm}$., larges de $2 \mathrm{~mm}$., sépales longs de $6 \mathrm{~mm}$. ; corolle longue de $15 \mathrm{~mm}$., son tube long de $10 \mathrm{~mm}$.

Madagascar : Analabe, au nord de Tananarive, dans la forêt à Mousses, sous bois, vers $\mathrm{I} .500 \mathrm{~m}$., arbuste de I- $2 \mathrm{~m}$. à fleurs roses (Perrier de la Bâthie I8.444).

\section{LE GROUPE SPÉCIFIQUE LINNÉEN DU CIRSIUM LANGEOLATUM (L.) HILL}

\section{par J. ARk̀NES}

Le Cirsium lanceolatum (L.) Hill. (sensu latissimo) constitue un groupe spécifique très polymorphe dont j'ai entrepris la révision en 1942 (I) par une étude critique sur sa sous-espèce crinitum (Boiss.) Ry; je me propose dans la présente note de préciser certains caractères généraux de l'espèce, d'en étudier en détail la distribution géographique actuelle et celle de ses sous-espèces ou variétés, d'en résumer l'organisation systéma-

(I) Cirsium lanceolatum (L) Hill subsp. crinitum (Boiss.) Ry. $-\mathrm{Ca}$,

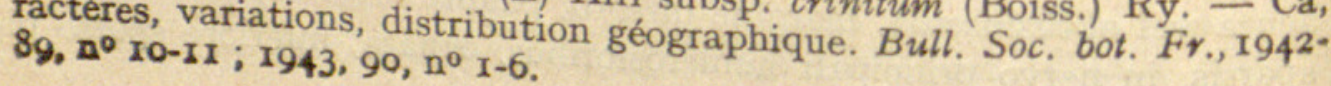




\section{$2 \mathrm{BHL}$ Biodiversity Heritage Library}

Benoist, R. 1945. "Descriptions de nouvelles Acanthacées malgaches." Notulae systematicae 12, 3-16.

View This Item Online: https://www.biodiversitylibrary.org/item/7377

Permalink: https://www.biodiversitylibrary.org/partpdf/168216

\section{Holding Institution}

Missouri Botanical Garden, Peter H. Raven Library

\section{Sponsored by}

Missouri Botanical Garden

\section{Copyright \& Reuse}

Copyright Status: In copyright. Digitized with the permission of the rights holder.

License: http://creativecommons.org/licenses/by-nc-sa/3.0/

Rights: https://biodiversitylibrary.org/permissions

This document was created from content at the Biodiversity Heritage Library, the world's largest open access digital library for biodiversity literature and archives. Visit BHL at https://www.biodiversitylibrary.org. 